\title{
Cardiovascular risk factors in a treatment-naïve, human immunodeficiency virus-infected rural population in Dikgale, South Africa
}

\author{
Felistas Mashinya, ${ }^{\text {a* }}$ Marianne Alberts, ${ }^{\text {a }}$ Robert Colebunders ${ }^{\mathrm{b}}$ and Jean-Pierre Van Geertruyden ${ }^{\mathrm{b}}$ \\ ${ }^{a}$ Department of Medical Science, Public Health and Health Promotion, School of Health Sciences, University of Limpopo \\ ${ }^{b}$ International Health Unit, Department of Epidemiology and Social Medicine, Faculty of Medicine, Antwerp University, Belgium \\ *Corresponding author:e-mail:mashinyaf@gmail.com
}

\begin{abstract}
Objective: The objective was to determine lipid levels and cardiovascular risk factors in treatment-naïve, human immunodeficiency virus (HIV)-infected rural African people in Limpopo province.

Design: This was a case control study.

Setting and subjects: The setting was Dikgale Health and Demographic Surveillance System Centre, Limpopo province. Treatment naïve, HIV-infected and HIV-negative people participated in the study.

Outcome measures: Demographic, lifestyle and chronic disease data were collected using the World Health Organization stepwise approach to surveillance (STEPS) questionnaire. Biochemical parameters were tested using standard biochemical methods. HIV testing and CD4 counts were performed using the Alere Determine ${ }^{\mathrm{TM}}$ HIV $1 / 2 \mathrm{Ag} / \mathrm{Ab}$ kit and The Alere Pima ${ }^{\mathrm{TM}}$ Analyser. Insulin resistance, low-density lipoprotein cholesterol (LDL cholesterol), and non-high-density lipoprotein cholesterol (non-HDL cholesterol) levels were calculated.

Results: The mean age of participants (years) was $49.7 \pm 16.6$. More HIV-infected than HIV-uninfected women consumed alcohol $(25.4 \%$ vs. $11.9 \%, p$-value $<0.05)$, and the prevalence of abdominal obesity was higher in HIV-uninfected than in HIV-infected women (74.6\% vs. 54.8\%, p-value < 0.05). Levels of total cholesterol (TC), HDL cholesterol, non-HDL cholesterol, LDL cholesterol and apolipoprotein A1 (ApoA1) were significantly lower in the HIV-infected than in the HIV-uninfected group. The prevalence of low HDL cholesterol was higher in HIV-infected than in HIV-uninfected people $(62.4 \%$ vs. $41.6 \%$, $p$-value $<0.01$ ). HIV infection increased the likelihood of low HDL cholesterol by 2.7 times ( $p$-value 0.001$)$. Male gender and alcohol use decreased the likelihood of low HDL cholesterol by $61 \%$ ( $p$-value 0.002 ) and $48 \%$ ( $p$-value 0.048 ), respectively. HIV infection was associated with low HDL cholesterol, ApoA1, LDL cholesterol and TC. Low CD4 count was associated with low body mass index, LDL cholesterol and high diastolic blood pressure.

Conclusion: The prevalence of cardiovascular risk factors was equally high in HIV-infected and in HIV-uninfected rural people, except for low HDL and alcohol consumption, which were significantly higher in HIV-infected people, while abdominal obesity was significantly higher in HIV-uninfected people. There is a need to raise awareness of cardiovascular risk factors in rural people in Limpopo province.
\end{abstract}

Keywords: abdominal obesity, alcohol, diabetes, hypertension, lipids

\begin{abstract}
Introduction
Human immunodeficiency virus (HIV) is one of the greatest worldwide public health challenges. An estimated 22.5 million people lived with HIV in sub-Saharan Africa in 2007, which comprised approximately $68 \%$ of global infection. ${ }^{1}$ Cardiovascular disease (CVD) is becoming a significant cause of morbidity and mortality in HIV-infected patients. ${ }^{2}$
\end{abstract}

HIV is a risk factor for CVD. ${ }^{3}$ It induces chronic inflammation that leads to several CVD-associated risk factors, such as type 2 diabetes, insulin resistance (IR), hypertension and dyslipidaemia, ${ }^{4-6}$ mediated by decreased adiponectin levels. ${ }^{6}$ Dyslipidaemia in HIV infection is characterised by decreased levels of total cholesterol (TC), low-density lipoprotein (LDL) cholesterol and high-density lipoprotein (HDL) cholesterol.7-10 Higher triglycerides (TG) levels were reported in advanced disease (acquired immune deficiency syndrome). ${ }^{11,12}$ Additionally, high levels of tobacco use, alcohol consumption and lower body mass index (BMI) are associated with CVD in HIV-infected people. ${ }^{13-15}$

Studies on the lipid profiles of treatment-naïve, HIV-infected people in South Africa show contradictory results on TC and TG levels, while HDL cholesterol and LDL cholesterol levels were reported by only one study.,16 Little is known of CVD risk factors in HIV-infected people in the rural province of Limpopo. The purpose of our study was to determine whether or not CVD risk factors were more elevated in HIV-infected than in HIV-uninfected rural people. We also determined associations between CVD risk factors and HIV and CD4 count. We searched for major predictors of low HDL cholesterol, an independent risk factor for CVD.

\section{Method \\ Study design and setting}

The study was a cross-sectional, nested, case control substudy of the main project, The Prevention, Control and Management of Chronic Disease project in a rural population, South Africa. The study was conducted in Dikgale Health and Demographic Surveillance System (HDSS) Centre, situated $50 \mathrm{~km}$ north east of Polokwane, the capital city of Limpopo province. There are approximately 35000 inhabitants in the Dikgale HDSS Centre, most of whom speak Northern Sotho.

\section{Study participants}

Eight hundred and fifteen randomly selected people participated in the main project. Participants who received pre-counselling were tested for HIV. Participants who tested 
HIV-positive and who were not on antiretroviral treatment according to the questionnaire formed the cases (89). The controls (178), matched for gender and age ( \pm 2 years), were randomly selected from those who tested negative for HIV.

Pregnant women were excluded from the study. Participants received information on the study prior to participation through door-to-door visits by trained fieldworkers and signed consent forms. Despite providing consent, some participants indicated that they did not want to know their results. Consenting participants were advised of participation dates, the local venue and times a week in advance. Participants received pre-counselling from trained counsellors on the scheduled date. The HIV results were revealed in postcounselling to participants who wanted to know their status. HIV-positive participants, and those with other abnormal biochemical abnormalities parameters, were referred to the local clinic or hospital for further analysis and management. Trained fieldworkers revisited participants and administered the questionnaire.

\section{Ethical considerations}

Permission for the study was sought from tribal chiefs. Ethical approval was obtained from the Ethics Committee of the University of Limpopo, Medunsa Campus. Written informed consent was obtained from participants and guardians of minors ( $<18$ years) prior to the study.

\section{Data collection}

The World Health Oganization (WHO) stepwise approach to surveillance (STEPS) questionnaire ${ }^{17}$ was used to obtain information on the medical condition of the subjects. Tuberculosis and HIV information was obtained using an additional questionnaire. The data collection was conducted from August 2011 to April 2012.

\section{Anthropometric measurements}

Weight was measured using Omron ${ }^{\circledR}$ BF 400 (Omron Healthcare, Kyoto, Japan). Subjects were asked to take off their shoes and heavy coats. Weight was measured to the nearest $0.1 \mathrm{~kg}$. Height was measured with a stadiometer. Barefoot subjects were asked to stand in an upright position. Height was measured to the nearest $0.1 \mathrm{~cm}$. BMI was calculated by dividing weight $(\mathrm{kg})$ by height $\left(\mathrm{m}^{2}\right)$. A BMI of $18.50-24.99 \mathrm{~kg} / \mathrm{m}^{2}$ was considered to be normal, $25-29.99 \mathrm{~kg} / \mathrm{m}^{2}$ overweight and $\geq 30 \mathrm{~kg} / \mathrm{m}^{2}$ obese. $^{18}$

Waist circumference (WC) and hip circumference (HC) were measured using a measuring tape. WC was measured around the area between the last rib and the hip bone, while $\mathrm{HC}$ was measured around the widest part in the gluteal area. Both parameters were measured to the nearest $0.1 \mathrm{~cm}$, and were used to calculate the waist to hip ratio.

\section{Blood pressure measurements}

Blood pressure (BP) was measured using the Omron ${ }^{\circledR}$ M5-1 (Omron Healthcare, Kyoto, Japan). The subjects were asked to sit and relax for five minutes before the first measurement was taken, and were restricted from talking during the measurement procedure. Three measurements were taken, with a few minutes' break inbetween. The mean of the last two values was calculated for systolic blood pressure (SBP) and diastolic blood pressure (DBP). High BP was defined as SBP above $140 \mathrm{mmHg}$ and/or DBP above $90 \mathrm{mmHg}^{19}$ and/or a selfreported history of antihypertensive drug use.

\section{Blood collection}

Fasting venous blood samples were drawn by registered nurses. Whole blood was used to measure CD4 count on the day of collection. Serum from clotted blood and plasma from whole blood were separated through centrifugation at $2000 \mathrm{rpm}$ for 15 minutes. Glucose and HIV status were analysed soon after centrifugation using plasma. The remaining samples were stored at $-80^{\circ} \mathrm{C}$ until analysis.

\section{Biochemical analysis}

Triglycerides (TGs), TC, HDL cholesterol, glucose and creatinine levels were determined on ILab ${ }^{\oplus} 300$ Plus Chemistry System (Instrumentation Laboratory Company, Milan, Italy). Insulin levels were measured on Beckman ${ }^{\circledast}$ Access Immunoassay System (Beckman Coulter, USA). Apolipoprotein B (ApoB), Apolipoprotein $A 1$ (ApoA1) and high-sensitivity C-reactive protein (hsCRP) levels were measured on the IMMAGE $^{\circledR}$ Immunochemistry System (Beckman Coulter, USA). Determine ${ }^{\mathrm{TM}}$ HIV-1/2 Ag/Ab Combo was initially used for HIV testing. Positive samples and $10 \%$ of negative samples were re-run using DoubleCheckGold ${ }^{\text {TM }}$ Ultra HIV $1 / 2$ kit. Both kits are Elisa ${ }^{\circledR}$-based and supplied by Inverness Medical, Tokyo, Japan. CD4 count was measured using the Pima ${ }^{\circ}$ Analyser (Inverness Medical, Tokyo, Japan). LDL cholesterol and IR were calculated using Friedewald ${ }^{20}$ and homeostatic model assessment-insulin resistance ${ }^{21}$ formulas, respectively. Non-HDL cholesterol was determined by subtracting the concentration of cholesterol in the HDL from that in the total plasma.

\section{Statistical analysis}

Statistical analysis was performed with Statistical Package for Social Science ${ }^{\circledast}$ version 20 . Variables were tested for normality using frequency histograms and line graphs. Data that were not normally distributed were logarithmically transformed for analysis. To make a comparison of group differences in respect of socio-demographic characteristics and lipid profiles, the independent Student's $t$-test was used for continuous variables and the chi-square test for categorical variables. Normally and not normally distributed data were presented as mean \pm standard deviation and median interquartile range, respectively. Bivariate correlation was used to establish associations between HIV, CD4 count and CVD-associated risk factors. Bivariate logistic regression was used to ascertain the individual influence of CVD-associated risk factors on HDL cholesterol levels. Multivariate stepwise forward and backward regression modelling was used to determine significant predictors of low HDL cholesterol levels.

\section{Results}

The mean age (years) of the participants was $49.7 \pm 16.6$. HIV-infected participants and HIV-uninfected participants had similar measurements in weight, height, BMI, WC, HC, hsCRP, insulin, DBP and SBP. Equally high prevalence rates of tobacco use ( $18 \%$ vs. $15.2 \%)$, IR ( $23.9 \%$ vs. $21.9 \%)$, hypertension $(42.7 \%$ vs. $45.5 \%)$ and diabetes mellitus ( $13.5 \%$ vs. $13.5 \%)$ were observed between the HIV-positive and HIV-negative participants, respectively. The prevalence rate of low HDL cholesterol was significantly higher in HIV-infected than in HIV-uninfected individuals $(62.4 \%$ vs. $41.6 \%$, $p$-value $<0.01)$. A significantly higher percentage of HIV-positive women than HIV-negative women consumed alcohol (25.4\% vs. $11.9 \%$, $p$-value $<0.05)$, and abdominal obesity was present in a significantly higher percentage of HIV-negative women (74.6\% vs. $54.8 \%, p$-value $<0.05)$, while their BMI remained below the threshold of 30 (Table 1). 
Table 1: Characteristics of human immunodeficiency virus-infected and human immunodeficiency virus-uninfected rural African people

\begin{tabular}{|c|c|c|c|c|c|c|}
\hline \multirow[b]{2}{*}{ Characteristics } & \multicolumn{2}{|c|}{ All participants } & \multicolumn{2}{|c|}{ Males } & \multicolumn{2}{|c|}{ Females } \\
\hline & $\begin{array}{c}\text { HIV-negative } \\
n=178\end{array}$ & $\begin{array}{c}\text { HIV-positive } \\
n=89\end{array}$ & $\begin{array}{c}\text { HIV-negative } \\
n=52\end{array}$ & $\begin{array}{c}\text { HIV-positive } \\
n=26\end{array}$ & $\begin{array}{c}\text { HIV-negative } \\
n=126\end{array}$ & $\begin{array}{c}\text { HIV-positive } \\
n=63\end{array}$ \\
\hline Age & $49.7 \pm 16.6$ & $49.7 \pm 16.8$ & $54.4 \pm 18.3$ & $55 \pm 18.8$ & $47.7 \pm 15.5$ & $47.6 \pm 15.6$ \\
\hline Weight (kg) & $68.3 \pm 15.7$ & $69.2 \pm 17.9$ & $66.4 \pm 15.3$ & $65.9 \pm 11$ & $69.1 \pm 15.8$ & $70.6 \pm 19.9$ \\
\hline Height (m) & $1.60 \pm 0.09$ & $1.62 \pm 0.09$ & $1.66 \pm 0.09$ & $1.68 \pm 0.09$ & $1.58 \pm 0.08$ & $1.60 \pm 0.08$ \\
\hline Body mass index & $26.2 \pm 6.5$ & $25.9 \pm 7.2$ & $23.7 \pm 5.4$ & $23.1 \pm 5.2$ & $27.2 \pm 6.7$ & $27 \pm 7.7$ \\
\hline WC $(\mathrm{cm})$ & $88.1 \pm 14.4$ & $85.5 \pm 16.5$ & $83.5 \pm 13.5$ & $80.9 \pm 10.7$ & $90.0 \pm 14.3$ & $87.3 \pm 18.1$ \\
\hline $\mathrm{HC}(\mathrm{cm})$ & $102.3 \pm 14.4$ & $100.8 \pm 13.5$ & $93.8 \pm 11.4$ & $97.3 \pm 7.4$ & $105.8 \pm 14.1$ & $102.1 \pm 15.2$ \\
\hline WC to HC & $0.87 \pm 0.12$ & $0.84 \pm 0.09 *$ & $0.90 \pm 0.15$ & $0.83 \pm 0.08^{*}$ & $0.85 \pm 0.11$ & $0.84 \pm 0.09$ \\
\hline $\mathrm{DBP}(\mathrm{mmHg})$ & $81.8 \pm 12.3$ & $81.1 \pm 15.8$ & $83.1 \pm 11.7$ & $80.0 \pm 13.2$ & $81.3 \pm 12.5$ & $81.6 \pm 16.8$ \\
\hline $\mathrm{SBP}(\mathrm{mmHg})$ & $129.5 \pm 21.6$ & $128.2 \pm 24.8$ & $136.0 \pm 22.1$ & $129.9 \pm 26.4$ & $126.8 \pm 20.9$ & $127.5 \pm 24.3$ \\
\hline Alcohol, $n(\%)$ & 35 (19.7) & $26(29.2)$ & $20(38.5)$ & $10(38.5)$ & $15(11.9)$ & $16(25.4)^{*}$ \\
\hline Tobacco use, $n(\%)$ & $27(15.2)$ & $16(18)$ & $17(32.7)$ & $8(30.8)$ & $10(7.9)$ & $8(12.7)$ \\
\hline $\mathrm{IR}_{,}^{*} n(\%)$ & 39 (21.9) & $21(23.9)$ & $13(25)$ & $8(30.8)$ & $26(20.6)$ & $13(21)$ \\
\hline Hypertension, ${ }^{* *} n(\%)$ & $81(45.5)$ & $38(42.7)$ & $29(55.8)$ & $10(38.5)$ & $52(41.3)$ & $28(44.4)$ \\
\hline Diabetes, $^{* * *} n(\%)$ & $24(13.5)$ & $12(13.5)$ & $6(11.5)$ & $5(19.2)$ & $18(14.3)$ & $7(11.1)$ \\
\hline Abdominal obesity, ${ }^{* * * *} n(\%)$ & $105(59)$ & $36(41.4)^{*}$ & $11(21.2)$ & $2(8)$ & $94(74.6)$ & $34(54.8)^{*}$ \\
\hline Low HDL cholesterol, ${ }^{* * * * *} n(\%)$ & $74(41.6)$ & $53(62.4)^{* *}$ & $10(19.2)$ & $14(53.8)^{* *}$ & $64(50.8)$ & $39(66.1)$ \\
\hline
\end{tabular}

DBP: diastolic blood pressure, HC: hip circumference, HDL: high-density lipoprotein, IR: insulin resistance, SBP: systolic blood pressure, WC: waist circumference

Values are mean \pm standard deviation, median interquartile range

${ }^{*} p$-value $<0.05,{ }^{* *} p$-value $<0.01$

* Insulin resistance $\geq 2.5$

** Hypertension or systolic blood pressure $>140 \mathrm{mmHg}$, diastolic blood pressure $>90 \mathrm{mmHg}$, and a history of high blood pressure

*** Diabetes glucose $>7 \mathrm{mmol} / \mathrm{l}$ and a history of diabetes

**** Abdominal obesity [waist circumference $>94 \mathrm{~cm}$ (males) and waist circumference $>80 \mathrm{~cm}$ (females)]

***** Low high-density lipoprotein cholesterol [high-density lipoprotein cholesterol $\leq 1.3 \mathrm{mmol} / \mathrm{l}$ (females), and high-density lipoprotein cholesterol $\leq 1.1 \mathrm{mmol} / \mathrm{l}$ (males)

Total cholesterol, HDL cholesterol, non-HDL cholesterol and LDL cholesterol levels were significantly lower ( $p$-value $<0.05$ ) in the HIV-infected than in the control group, while no statistical difference was observed between TG levels (Table 2).

The pattern persisted in males, while LDL cholesterol and nonHDL cholesterol levels were not significantly different between HIV-positive and HIV-negative females. ApoB levels were not significantly different in HIV-positive than in HIV-positive people. ApoA1 levels were significantly lower in the HIVpositive group than in the control group. Glucose levels were significantly lower in HIV-infected than in the control group
( $p$-value 0.04). Glucose levels remained significantly lower ( $p$-value 0.01 ) in females, while no significant difference was observed in males.

HIV infection significantly correlated negatively with HDL cholesterol $(r=-0.23, p$-value $<0.00)$, ApoA1 $(r=-0.24$, $p$-value 0.00$), \mathrm{LDL}$ cholesterol $(r=-0.18, p$-value 0.00$)$ and TC $(r=-0.23, p$-value 0.00$)$, while CD4 count [only available for 52 participants (mean value 397 cells $/ \mu \mathrm{l}$ )] significantly correlated positively with BMI $(r=0.37, p$-value 0.00$)$ and LDL cholesterol $(r=0.30, p$-value 0.03$)$, but negatively with DBP $(r=-0.43$, $p$-value 0.00 ) (Table 3 ).

Table 2: Biochemical characteristics of human immunodeficiency virus-infected and human immunodeficiency virus-uninfected rural African people

\begin{tabular}{|c|c|c|c|c|c|c|}
\hline \multirow[b]{2}{*}{ Characteristics } & \multicolumn{2}{|c|}{ All participants } & \multicolumn{2}{|c|}{ Males } & \multicolumn{2}{|c|}{ Females } \\
\hline & $\begin{array}{c}\text { HIV-negative } \\
n=178\end{array}$ & $\begin{array}{c}\text { HIV-positive } \\
n=89\end{array}$ & $\begin{array}{c}\text { HIV-negative } \\
n=52\end{array}$ & $\begin{array}{c}\text { HIV-positive } \\
n=26\end{array}$ & $\begin{array}{c}\text { HIV-negative } \\
n=126\end{array}$ & $\begin{array}{c}\text { HIV-positive } \\
n=63\end{array}$ \\
\hline $\mathrm{hsCRP}(\mathrm{mg} / \mathrm{l})$ & $2.32(0.88-6.09)$ & $1.87(0.68-5.64)$ & $1.86(0.87-3.17)$ & $3.79(0.93-10.03)$ & $2.81(0.88-6.99)$ & $1.58(0.61-5.23)$ \\
\hline Glucose (mmol/l) & $5.79 \pm 2.78$ & $5.22 \pm 1.63^{*}$ & $5.29 \pm 0.93$ & $5.51 \pm 2.13$ & $6.00 \pm 3.23$ & $5.11 \pm 1.38^{*}$ \\
\hline Insulin (ulU/ml) & $6.28(3.27-9.42)$ & $4.91(2.56-9.33)$ & $5.80(2.74-9.63)$ & $4.85(2.37-15.20)$ & $6.37(3.43-9.43)$ & $4.91(3.12-9.10)$ \\
\hline $\mathrm{TG}(\mathrm{mmol} / \mathrm{l})$ & $1.18(0.83-1.70)$ & $1.12(0.78-1.67)$ & $1.29(0.87-1.74)$ & $1.03(0.74-2.15)$ & $1.14(0.79-1.70)$ & $1.15(0.78-1.52)$ \\
\hline $\mathrm{TC}(\mathrm{mmol} / \mathrm{l})$ & $4.74 \pm 1.15$ & $4.16 \pm 1.27^{*}$ & $4.74 \pm 1.00$ & $3.92 \pm 1.02^{*}$ & $4.75 \pm 1.21$ & $4.27 \pm 1.35^{*}$ \\
\hline LDL cholesterol (mmol/l) & $2.76 \pm 0.95$ & $2.37 \pm 1.10^{*}$ & $2.69 \pm 0.85$ & $2.15 \pm 1.03^{*}$ & $2.79 \pm 0.99$ & $2.47 \pm 1.12$ \\
\hline HDL cholesterol (mmol/l) & $1.37 \pm 0.35$ & $1.17 \pm 0.44^{*}$ & $1.39 \pm 0.33$ & $1.07 \pm 0.36^{*}$ & $1.36 \pm 0.36$ & $1.21 \pm 0.46^{*}$ \\
\hline Non-HDL cholesterol (mmol/l) & $3.38 \pm 1.05$ & $2.99 \pm 1.10^{*}$ & $3.36 \pm 0.94$ & $2.85 \pm 0.98^{*}$ & $3.39 \pm 1.10$ & $3.05 \pm 1.15$ \\
\hline ApoB (mg/dl) & $89.0 \pm 27.9$ & $83.9 \pm 24.3$ & $85.8 \pm 26.3$ & $78.8 \pm 26.7$ & $90.3 \pm 28.5$ & $86.3 \pm 23.0$ \\
\hline ApoA1 (mg/dl) & $150.5 \pm 32$ & $133.4 \pm 33.3^{*}$ & $153.4 \pm 33.3$ & $121.8 \pm 25.8^{*}$ & $149.3 \pm 31.4$ & $138.5 \pm 35.3^{*}$ \\
\hline
\end{tabular}

ApoA1: apolipoprotein A1, ApoB: apolipoprotein B, HDL: high-density lipoprotein, hsCRP: high-sensitivity C-reactive protein, LDL: low-density lipoprotein, TC: total cholesterol, TG: triglycerides

Values are presented as mean \pm standard deviation, median interquartile range

* $p$-value $<0.05$

Cut-offs: Low-density lipoprotein cholesterol $<3 \mathrm{mmol} / \mathrm{l}$, total cholesterol $<5 \mathrm{mmol} / \mathrm{l}$, and triglycerides $<1.7 \mathrm{mmol} / \mathrm{l}$ 
Table 3: Bivariate correlations of cardiovascular disease-associated factors with human immunodeficiency virus and CD4 count

\begin{tabular}{|c|c|c|c|c|c|c|}
\hline & HDL cholesterol & ApoA1 & LDL cholesterol & $\mathrm{TC}$ & BMI & DBP \\
\hline HIV & $-0.239^{*}$ & $-0.240^{*}$ & $-0.179 *$ & $-0.225^{*}$ & -0.02 & -0.02 \\
\hline CD4 count & -0.04 & -0.21 & $0.303^{* *}$ & 0.25 & $0.370 *$ & $-0.43^{*}$ \\
\hline
\end{tabular}

ApoA1: Apolipoprotein A1, BMI: body mass index, DBP: diastolic blood pressure, HDL: high-density lipoprotein, HIV: human immunodeficiency virus, LDL: low-density lipoprotein, TC: total cholesterol

Values are correlation coefficients

${ }^{*} p$-value $<0.01,{ }^{* *} p$-value $<0.05$

The current study focused on HDL cholesterol levels as the latter is regarded as a significant independent risk factor for CVD. ${ }^{2,22-24}$ Bivariate analysis showed that an HIV-positive person is 2.3 times more likely to have low HDL cholesterol than an HIVnegative person (Table 4). HIV infection increased the likelihood of low HDL cholesterol by 2.7 times in a multivariate analysis. Overall, HIV-positive status, gender and alcohol consumption were significant predictors of low HDL cholesterol in this study.

\section{Discussion}

The mean age (years) of the participants was $49.7 \pm 16.6$. There was no significant difference in mean anthropometric values, BP measurements and insulin levels between HIV-positive and HIV-negative people. These similarities may indicate that our study population was in the early to mid stage of HIV infection, which was confirmed by the mean CD4 count of nearly 400 cells $/ \mu$ l. Similarities in BMI, WC and HC between treatmentnaïve HIV-infected and HIV-uninfected people have also been observed elsewhere. ${ }^{25}$

The prevalence rates of diabetes mellitus, hypertension and IR were not different between HIV-positive and HIV-negative males and females. However, overall, the prevalence rates were high, putting both HIV-positive and HIV-negative people at risk of CVD occurrence.

Similar prevalence rates of diabetes mellitus between treatment-naïve HIV-infected and HIV-uninfected women were reported in a menopause study, ${ }^{25}$ and are consistent with those found in the current study. Higher prevalence rates of diabetes mellitus were reported in HIV-uninfected than in HIV-infected men $(12 \%$ vs. $7 \%, p$-value $<0.0001)$ by Womack et al. ${ }^{26}$ Several hypothesis have been made about the relationship between HIV and diabetes, including the chronic inflammation state, leading to decreased adiponectin levels and IR, genetic susceptibility, obesity prevalence, sedentariness and the use of opiates. $4,25,27 \mathrm{~A}$ significant difference in the hypertension prevalence rate between HIV-infected and HIVuninfected people was not found in this study. Similar results were reported by Baekken et al. ${ }^{28}$ Other investigators found a significantly higher prevalence rate of hypertension in HIVuninfected women relative to that in HIV-infected women $(31.4 \%$ vs. $20.1 \%, p$-value $<0.001)$, while no significant difference was observed in men. ${ }^{29}$ These inconsistencies could relate to differences in HIV duration and different populations. ${ }^{5}$

Abdominal obesity was more predominant in HIV-negative than in HIV-positive females (59\% vs. $41.1 \%, p$-value < 0.05 ). Although this study did not include a dietary analysis, earlier reports found that the Dikgale HDSS community consumed high carbohydrates and low-fat, low-dietary fibre which may favour the development of abdominal obesity. ${ }^{30}$ Similarly, a study conducted in a South African rural area showed obesity to be significantly more common in HIV-uninfected than in HIV-infected adults ( $24.5 \%$ vs. $20 \%, p$-value $<0.001)$, and this difference was largely contributed by women (34\% HIVuninfected vs. $23.8 \%$ HIV-infected), while obesity rates were not significantly different in HIV-infected and HIV-uninfected men. ${ }^{29}$

A significantly higher prevalence rate of HIV-infected than HIVuninfected females consumed alcohol $(25.4 \%$ vs. $11.9 \%, p$-value $<0.05$ ), while similar rates were observed in HIV-infected and HIV-uninfected males. Dikgale HDSS is a poor rural community, and poverty is a driver of commercial sex work for most women, exposing them to the sale of alcohol and the risk of HIV infection. Significantly higher alcohol use by HIV-positive than HIV-negative women has been reported elsewhere. ${ }^{31}$ The current study observed similar tobacco usage in HIV-positive and HIV-negative males and females. Higher tobacco use in HIVpositive than in HIV-negative people was reported elsewhere. ${ }^{32}$

Table 4: Predictors of low high-density lipoprotein cholesterol levels in participants in the Dikgale Health and Demographic Surveillance System Centre $(n=267)$

\begin{tabular}{lccc}
\hline \multirow{2}{*}{ Predictors } & \multicolumn{2}{c}{ Bivariate logistic regression } & \multicolumn{2}{c}{ Multivariate logistic regression } \\
\cline { 2 - 4 } HIV-positive & OR $(95 \% \mathrm{Cl})$ & $p$-value & Adjusted OR (95\% Cl) \\
Male & $2.33(1.37-3.96)$ & 0.002 & $2.69(1.52-4.75)$ \\
Alcohol & $0.35(0.20-0.62)$ & $<0.001$ & $0.39(0.22-0.71)$ \\
WC obesity & $0.49(0.27-0.90)$ & 0.02 & $0.52(0.27-0.99)$ \\
IR & $1.63(1-2.66)$ & 0.05 & 0.047 \\
hsCRP & $1.21(0.67-2.16)$ & 0.53 & \\
Hypertension & $2.07(0.97-4.40)$ & 0.06 & \\
Smoking & $0.91(0.56-1.48)$ & 0.71 & \\
Diabetes & $0.48(0.24-0.96)$ & 0.04 & 0.49 \\
\hline
\end{tabular}

$\mathrm{Cl}$ : confidence interval, HIV: human immunodeficiency virus, hsCRP: C-reactive protein, IR: insulin resistance, OR: odds ratio, WC: waist circumference Values are unadjusted odds ratio (95\% confidence interval) from bivariate logistic regression, and adjusted odds ratio (95\% confidence interval) from multivariate stepwise forward and backward logistic regression modelling 
The lipid profile in HIV-infected people (89) was altered, compared to that in HIV-uninfected people (Table II). Our study showed significant decreases in TC, LDL cholesterol and HDL cholesterol in HIV-positive people compared to that in HIV-negative people. This lipoprotein pattern is associated with the potential risk of premature CVD developing. ${ }^{33}$ The dyslipidaemia caused by the effects of the virus itself results from the inflammatory cytokine response to HIV infection. ${ }^{34,35}$

Several studies have reported similar decreases in TC, HDL cholesterol and LDL cholesterol, ${ }^{36-39}$ while higher LDL cholesterol levels have been reported elsewhere. ${ }^{40-42}$ Interestingly, a study ${ }^{16}$ conducted on urban South African women reported no significant difference in TC between treatment-naïve HIV-positive and HIV-negative women, while HDL cholesterol and LDL cholesterol were not reported. The current study observed no significant difference in TG levels between HIV-positive and HIV-negative people for the whole group, and for males and females separately. Some studies have demonstrated disparate TG results. ${ }^{9,38,42}$ Variations in TG levels were attributed to the difference in degree of immunosuppression, with TG levels escalating with an increase in immunosuppression. ${ }^{11,43}$

Consistent with the current study, Baker et $\mathrm{al}^{44}$ reported no significant association between HDL cholesterol and CD4 count. By contrast, two earlier studies by El-Sadr et $\mathrm{al}^{45}$ and Rose et al ${ }^{46}$ reported significant associations between $\mathrm{HDL}$ cholesterol and CD4 count.

Even though the contribution of HIV to lipid abnormalities may be difficult to distinguish from that of classical cardiovascular risk factors, in the current study, after controlling for the effect of each risk factor, the multivariate regression model indicated HIV infection, male gender and alcohol use as significant predictors of low HDL cholesterol. Having 2.8 times more likelihood of low HDL cholesterol in HIV-infected people therefore increases the risk of CVD in this HIV-infected rural population. Similarly, Oka et al ${ }^{12}$ reported an association between HIV disease and lipid metabolism in a Japanese male population.

The strength of this study was that standardised techniques were used, which included the WHO STEPS questionnaire and the repeated measurement of BP and HIV, as well as the use of controls in analysing the biochemical parameters.

Limitations of the study included the relatively small number of study subjects. Moreover, the sample largely comprised people living at home, thus it was skewed towards an older sedentary group. Migrant workers and people with employment were away from home when the study was conducted. This could have biased the results. A similar study is needed on the migrant population. Information on smoking and alcohol use was obtained using the WHO STEPS questionnaire, considered to be a reliable instrument.

\section{Conclusion}

The treatment-naïve, HIV-infected rural population in the Dikgale HDSS had significantly lower levels of TC, LDL cholesterol, HDL cholesterol and ApoA1, but not TG, than an HIV-uninfected rural population. The study also revealed a high prevalence of CVD risk factors, such as hypertension, diabetes, $I R$, tobacco use and alcohol consumption in treatment-naïve, HIV-infected people, which confirms an earlier report on the general population. ${ }^{47}$ To our knowledge, this study is the first to have reported on CVD risk factors in treatment-naïve,
HIV-infected people in the Limpopo province. HIV outreach programmes should focus more on the management of noncommunicable diseases in rural areas where elderly people reside. Further studies on subclasses of lipoproteins may provide more in-depth knowledge of the risk of CVD in this rural population.

\section{Conflict of interest - The authors declare no conflict of interest.}

Acknowledgements - Acknowledgement is given to the fieldworkers and Dikgale HDSS team members for recruitment of the subjects and collection of the data. Gratitude is also extended to the community at Dikgale HDSS site for their collaboration during this study. We are grateful for the support of the Belgium Development Co-operation through Vlaamse Interuniversitaire Raad-University Development Cooperation (VLIR-UOS) within the framework of an institutional collaboration between the University of Limpopo and Flemish universities.

\section{References}

1. Joint United Nations Programme on HIV/AIDS, World Health Organization. AIDS epidemic update. UNAIDS [homepage on the Internet]. c2012. Available from: http://data.unaids.org/pub/ epislides/2007/2007_epiupdate_en.pdf

2. Duprez DA, Kuller LH, Tracy R, et al. Lipoprotein particle subclasses, cardiovascular disease and HIV infection. Atherosclerosis. 2009;5(1):1-6.

3. Baker JV, Lundgren JD. Cardiovascular implications from untreated human immunodeficiency virus infection. Eur Heart J. 2011;32(8):945-951.

4. Reid MJA, Tsima BM, Kirk B. HIV and diabetes in Africa. African Journal of Diabetes Medicine. 2012; 20(2):28-32.

5. Manner IW, Baekken M, Oektedalen O, et al. Effects of HIV duration on ambulatory blood pressure in HIV-infected individuals with high office blood pressure. Blood Press. 2010;19(3):188-195.

6. Palios J, Kadoglou NPE, Lampropoulous S. The pathophysiology of HIV/HAART-related metabolic syndrome leading to cardiovascular disorders: the emerging role of adipokines. Exp Diabetes Res. 2012;2012:103063.

7. Rose H, Hoy J, Woolley I, et al. HIV infection and high density lipoprotein metabolism. Atherosclerosis. 2008;199(1):79-86.

8. Baker JW, Ayenew H, Quick KH, et al. High-density lipoprotein particles and markers of inflammation and thrombotic activity in patients with untreated HIV infection. J Infect Dis. 2010;201(2):285-292.

9. Fourie CMT, Van Rooyen JM, Kruger A, Schutte AE. Lipid abnormalities in a never-treated HIV-1 subtype C-infected African population. Lipids. 2010;45(1):73-80.

10. Oka F, Naito T, Oike M, et al. Correlation between HIV disease and lipid metabolism in anti-retroviral naïve HIV infected patients in Japan. J Infect Chemother. 2011;18(1)17-21.

11. Grunfeld C, Pang M, Doerrler W, et al. Lipids, lipoproteins, triglyceride clearance and cytokines in human immunodeficiency virus infection and the acquired immunodeficiency syndrome. J Clin Endocrinol Metab. 1992;74(5):1045-1052.

12. Feingold KR, Krauss RM, Pang M. The hypertriglyceridemia of acquired immunodeficiency syndrome is associated with an increased prevalence of low density lipoprotein subclass B. J Clin Endocrinol Metab. 1993;76(6):1423-1427.

13. Neuman T, Reinsch N, Esser $S$, et al. Smoking behaviour of HIV-infected patients. Health. 2010;2(8):913-918.

14. Freiberg MS, McGinnis KA, Kraemer K, et al. The association between alcohol consumption and prevalent cardiovascular diseases among HIV infected and uninfected men. J Acquir Immune Defic Syndr. 2010;53(2): 247-253.

15. Lemmer CE, Badri M, Visser M, Mayosi BM. A lower body mass index is associated with cardiomyopathy in people with HIV infection: evidence from a case comparison study. S Afr Med J. 2011;101(2):1-4. 
16. Hattingh Z, Walsh C, Veldman FJ, Bester CJ. The metabolic profiles of HIV-infected and non-infected women in Mangaung, South Africa. S Afr J Clin Nutr. 2009;22(1):23-28.

17. World Health Organization. Chronic diseases and health promotion: STEPwise approach to surveillance (STEPS). WHO [homepage on the Internet]. c2013. Available from: http://www.who.int/chp/steps/en/

18. World Health Organization. Physical status: the use and interpretation of anthropometry. Geneva: WHO; 1995.

19. Federation R. Risk factor: blood pressure. World Health Organization [homepage on the Internet]. c2012. Available from: http://www. who.int/cardiovascular_diseases/en/cvd_atlas_05_HBP.pdf

20. Friedewald WT, Levy RI, Fredrickson DS. Estimation of the concentration of low-density lipoprotein cholesterol in plasma, without use of the preparative ultracentrifugation. Clin Chem. 1972;18(6):499-502

21. Matthews DR, Hosker JP, Rudenski AS, et al. Homeostasis model assessment: insulin resistance and beta-cell function from fasting plasma glucose and insulin concentrations in man. Diabetologia. 1985;28(7):412-419.

22. Asztalos BF, Schaefer EJ. HDL in atherosclerosis: actor or bystander? Atheroscler Suppl. 2003;4(1):21-29.

23. Jericho C, Knobel H, Sorli ML, et al. Prevalence of cardiovascular risk factors in HIV-infected patients. Rev Clin Esp. 2006;206(11):556-559.

24. Di Angelantonio E, Sarwar N, Perry P, et al. Major lipids, apolipoproteins and risk of vascular disease. JAMA. 2009;302(18):1993-2000.

25. Howard AA, Floris-Moore M, Arnsten JH, et al. Disorders of glucose metabolism among HIV-infected women. Clin Infect Dis. 2005;40(10):1492-1499.

26. Womack JA, Goulet JL, Gibert C, et al. Increased risk of fragility fractures among HIV infected compared to uninfected male veterans. PLoS One. 2011;6(2):e17217

27. Samaras K. Prevalence and pathogenesis of diabetes mellitus in HIV-1 infection treated with combined antiretroviral therapy. J Acquir Immune Defic Syndr. 2009;50(5):499-505.

28. Baekken M, Os I, Sandvik L, Oektedalen O. Hypertension in an urban HIV positive population compared with the general population: influence of combination anti-retroviral therapy. J Hypertens. 2008;28(11):2126-2133.

29. Malaza A, Mossong J, Barnighausen T, Newell M. Hypertension and obesity in a high HIV prevalence rural area in South Africa. PloS One. 2012;7(10):1-6.

30. Steyn NP. Nutrition and chronic diseases of lifestyle in South Africa. South African Medical Research Council [homepage on the Internet]. c2013. Available from: http://www.mrc.ac.za/chronic/ cdlchapter4.pdf

31. Figueroa-Cosme WI, Lopez-Cordova NM, Capriles-Qoiros JA. Mothers of adolescents girls: comparing HIV positive and HIV negative women. Ethn Dis. 2010;20(1 Suppl 1):S1-S127.
32. Crothers K, Goulet JL, Rodriguez-Barradas MC, et al. Impact of cigarette smoking on mortality in HIV-positive and HIV negative veterans. AIDS Educ Prev. 2009;21(3 Suppl):40-53.

33. Anastos K, Ndamage F, Lu D, et al. Lipoprotein levels and cardiovascular risk in HIV infected and uninfected Rwandan women. AIDS Res Ther. 2010;7:34.

34. Oh J, Hegele RA. HIV-associated dyslipidaemia pathogenesis and treatment. Lancet Infec Dis. 2007;7(12):787-796.

35. Inankur A, Nicholls SJ, Jahangiri A. High density lipoprotein: is the good cholesterol turning bad? Curr Cardiovasc Risk Rep. 2011;5:18-28.

36. Badiou S, De Boever CM, Dupuy AM, et al. Decrease in LDL size in HIV-positive adults before and after lopinavir/ritonavircontaining regimen: an index of atherogenicity? Atherosclerosis. 2003;168(1):107-113.

37. Riddle SA, Smit E, Cole SR, et al. Impact of HIV infection and HAART on serum lipids in men. JAMA. 2003;289(22):2978-2982.

38. Oduola T, Akinbolade AA, Oladokun LO, et al. Lipid profiles in people living with HIV/AIDS on ARV therapy in an urban area of Osun State, Nigeria. World J Med Sci. 2009;4:18-21.

39. Nguemaim NF, Mbuagbaw J, Nkoa T, et al. Serum lipid profiles in highly active antiretroviral therapy-naïve HIV-infected patients in Cameroon: a case-control study. HIV Med. 2010;11(6): 353-359.

40. Tien PC, Schneider MF, Cox C, et al. HIV, HAART, and lipoprotein particle concentrations in the Women's Interagency HIV Study. AIDS. 2010;24(18):2809-2817.

41. Adewole OO, Eze S, Betiku Ye, et al. Lipid profile in HIV/AIDS patients in Nigeria. Afr Health Sci. 2010;10(2):144-149.

42. Kumar A, Sathian B. Assessment of lipid profile in patients with human immunodeficiency virus (HIV/AIDS) without antiretroviral therapy. Asian Pacific Journal of Tropical Disease. 2011:24-27.

43. Armstrong C, Liu E, Grispoon S, et al. Dyslipidemia in an HIV-positive, antiretroviral treatment-naïve population in Dar es Salaam, Tanzania. J Acquir Immune Defic Syndr. 2011;57(2):141-145.

44. Baker JV, Neuhaus J, Duprez D, et al. Inflammation predicts changes in high-density lipoprotein particles and apolipoprotein A1 following initiation of antiretroviral therapy. AIDS. 2011;25(17):2133-2142.

45. El-Sadr WM, Mullin CM, Gibert C, et al. Effects of HIV disease on lipid, glucose and insulin levels: results from a large antiretroviral-naïve cohort. HIV Med. 2005;6(2):114-121.

46. Rose H, Woolley I, Hoy J, et al. HIV infection and high density lipoprotein: the effect of the disease vs the effect of treatment. Metabolism. 2006;55(1):90-95.

47. Alberts M, Urdal P, Steyn K, et al. Prevalence of cardiovascular diseases and associated risk factors in a rural black population of South Africa. Euro J Cardiovasc Prev and Rehabil. 2005;12(4):347-354.

Received: 02-04-2013 Accepted: 06-07-2013 